ANAP Brasil $\quad \begin{aligned} & \text { ISsN } 1984-3240 \\ & \text { v. 9, n. } 17\end{aligned}$

\title{
Estudo do Teor de Metacaulim em Concretos de Alto Desempenho
}

Study the Percentage of Metakaolin in High-Performance Concretes

Estudio de los Niveles de Metacaolín en Hormigones de Alto Rendimiento

\section{Manuela Mendes Souza}

Graduando, UNESP, Brasil.

manuelamendessouza@gmai.com

Tiago Armando Camara

Graduando, UNESP, Brasil.

tiagocamara14@gmail.com

Jorge Luis Akasaki

Professor Doutor, UNESP, Brasil.

jorge.akasaki@gmail.com 


\section{RESUMO}

O Concreto de Alto Desempenho (CAD), em grande ascensão no mundo, é alvo de pesquisas e empresas que necessitam de produtos com melhor qualidade e desempenho. Este projeto tem por finalidade indicar a porcentagem ideal para substituição de parte do cimento por metacaulim e a melhor dosagem dos materiais para a obtenção do CAD. O método de dosagem utilizado foi baseado no IPT/EPUSP. Utilizou-se um traço de 1:4,68, teor de argamassa de $46,7 \%$, relação água/aglomerante de 0,31 . A quantidade de aditivo superplastificante variou para cada traço devido a busca pela consistência adequada definida pelo Slump Test, sendo a altura de abatimento de $20 \pm 2 \mathrm{~cm}$. Parte do cimento foi substituído por metacaulim nas proporções de $5,10,15$ e $20 \%$ em relação à massa de cimento, e foi adicionado sílica ativa na proporção de $8 \%$ da massa total de aglomerante. Para cada traço confeccionou-se um traço controle sem adição de metacaulim. Os ensaios de resistência à compressão e à tração e o de módulo de elasticidade foram realizados nas idades de 7, 28, 60 e 90 dias e os ensaios de absorção de água foram realizados na idade 28 dias. Os ensaios foram realizados conforme as normas indicadas pela Associação Brasileira de Normas Técnicas (ABNT). Com os resultados obtidos pode-se afirmar que o teor de substituição de $10 \%$ obteve os melhores desempenhos comparados com o CAD referência, mostrando que a substituição de parte do cimento por metacaulim melhora as propriedades mecânicas do CAD.

PALAVRAS-CHAVE: Concreto de alto desempenho. Metacaulim. Resistência à compressão.

\section{ABSTRACT}

The High-Performance Concrete (HPC), in great rise in the world, is target of researches and companies that need products with best quality and performance. This project is intended to indicate the percentage for substitution of the cement by metakaolin, and the best dosage of material for obtaining HPC. The dosage method used was based on IPT/EPUSP. Used a mixture of 1:4,68, mortar percentage of $46,7 \%$, water/binder relation of 0,31 . The amount of superplasticizer varied for each mixture because the search for the proper consistency defined by Slump Test, and the rebate height of $20 \pm 2 \mathrm{~cm}$. Part of the cement was substituted by metakaolin in proportions of 5, 10, 15 e $20 \%$ relative to cement weight, and silica fume was added in proportion of $8 \%$ of the total mass of binder. For each mixture was made a control mixture without adding metakaolin. The compressive and tensile strength tests and elasticity modulus were made at the ages of 7, 28, 60 and 90 days. The tests were performed according to the standards set by the Brazilian Association of Technical Standards (ABNT). The result showed that the substitution of $10 \%$ had the best performance compared with the reference HPC, showing that the substitution of the cement by the metakaolin improves the mechanical properties of the HPC.

KEY-WORDS: High-Performance Concrete. Metakaolin. Compressive strength.

\section{RESUMEN}

El Hormigón de Alto Rendimiento (HAR), un gran aumento en el mundo, es el objeto de la investigación y las empresas que necesitan productos con la mejor calidad y rendimiento. Este diseño tiene por objetivo indicar el porcentaje ideales mediante la sustitución de parte del cemento con metacaolín, y la mejor dosificación de los materiales para la obtención de HAR. El método de ensayo utilizado se basó en IPT/EPUSP. Se utilizó una mezcla de $1: 4,68$, el contenido del mortero del $46,7 \%$, el agua/aglomerante 0,31 . La cantidad de superplastificante variado para cada mezcla debido a la búsqueda de consistencia adecuada definida por Slump Test, y la altura de reembolso de $20 \pm 2 \mathrm{~cm}$. Parte de cemento fue reemplazado por metacaolín en las proporciones de 5, 10, 15 y $20 \%$ con respecto al peso de cemento, y se añadió humo de sílice en la proporción de $8 \%$ del peso total de aglomerante. Para cada mezcla fue producido una mezcla de control sin la adición de metacaolín. La resistencia a la tracción pruebas de compresión y el módulo elástico se realizaron a las edades de 7, 28, 60 y 90 días y los ensayos de absorción de agua se llevaron a cabo a la edad de 28 días. Las pruebas se realizaron de acuerdo con las normas establecidas por la Asociación Brasileña de Normas Técnicas (ABNT). Con los resultados obtenidos se puede decir que la sustitución del contenido de $10 \%$ logra el mejor rendimiento en comparación con el HAR de referencia, que muestra que la sustitución del cemento por metacaolín mejora las propiedades mecánicas de la HAR.

PALABRAS CLAVE: Hormigón de alto rendimiento. Metacaolín. Resistencia a la compresión. 


\section{INTRODUÇÃO}

A área da construção civil vem crescendo consideravelmente há algum tempo no mundo, com construções mais complexas, sofisticadas e que exigem cada vez mais da engenharia. Tal realidade implica em um consumo crescente de concreto, constituído de agregado graúdo, agregado miúdo, água, cimento, adições e aditivos. Essa demanda fez com que o cimento Portland se tornasse o segundo material de maior consumo no mundo, perdendo apenas para a água, sendo assim o material estrutural e de construção civil mais consumido, segundo Valverde (2001).

O Sindicato Nacional da Indústria do Cimento (SNIC) analisa o consumo, a produção, e exportação e importação de cimento no Brasil. Segundo o SNIC, o Brasil produziu 36 milhões de toneladas de cimento em 2004, e em 2013 esse número aumentou para 70,2 milhões de toneladas, mostrando um crescimento de $95 \%$ em relação a 2004.

O SNIC também analisou o consumo total de cimento no Brasil de 1950 a 2013 e mostrou que o número só aumentou a cada ano. Ainda segundo o SNIC, em 1950 o consumo total foi de 1,8 milhões de toneladas e em 2013 esse número subiu para 71 milhões de toneladas, revelando um aumento de cerca de 40 vezes.

Com a crescente evolução das exigências de mercado com relação ao desempenho estrutural dos projetos arquitetônicos e de engenharia, tornou-se necessário a produção de concretos com resistências superiores à do concreto convencional. O Concreto de Alto Desempenho (CAD) atende a essas necessidades.

Duas das mais desenvolvidas e poderosas sociedades atuais, os Estados Unidos e o Canadá, consideram o investimento no estudo das estruturas de concreto como um dos mais importantes investimentos na ciência e tecnologia para obter e manter a qualidade de vida de seu povo e a liderança de seu parque industrial. Essas sociedades entendem que o profundo conhecimento sobre concreto posiciona e mantém a sua indústria na fronteira do conhecimento, assegurando sua alta competitividade (HELENE e ANDRADE, 2007).

Um outro ponto a ser destacado, porém não de menor importância, é que esse aumento na produção de concreto tem causado sérios problemas ambientais em todo o mundo. A queima da matéria prima libera grandes quantidades de $\mathrm{CO}_{2}$, poluindo o ar, e também, a obtenção dessa matéria prima em larga escala gera degradação ambiental.

Na produção do concreto são gerados resíduos que causam impactos ambientais negativos. Estima-se que cerca de $50 \%$ dos resíduos sólidos gerados pelo conjunto das atividades humanas venham da construção civil conforme consta no site do MMA (MINISTÉRIO DO MEIO AMBIENTE). Segundo Química Ensinada (2011), a indústria do cimento, a qual produz um dos constituintes mais importantes do concreto, é responsável pela emissão de 5 a $7 \%$ da emissão mundial de dióxido de carbono. No Brasil, para cada tonelada de clínquer produzido, que é a 
massa crua utilizada durante a fabricação do cimento, são liberados na atmosfera cerca de 700 $\mathrm{Kg}$ de $\mathrm{CO}_{2}$, disponível em Química Ensinada (2011).

Segundo Toledo Filho et al. (2002), o processo de fabricação do cimento Portland requer grandes quantidades de energia. Ainda conforme Toledo Filho et al. (2002), as indústrias de cimento são responsáveis por cerca de 7\% da emissão mundial de gás carbônico na atmosfera, o que contribui para o aumento do aquecimento global. Segundo Aitcin (2000), o processo de evolução do concreto aconteceu a partir de 1960, quando os concretos chegavam à resistência de $53 \mathrm{MPa}$ e em 1970 já eram produzidos com até $70 \mathrm{MPa}$. Ultrapassar esses limites só foi possível com o surgimento de novos materiais.

Assim, para que fosse possível obter melhores qualidades no concreto, como o aumento da resistência, foi necessário o desenvolvimento de estudos da adição de minerais e aditivos, que culminou na descoberta da ação dos aditivos superplastificantes. Tal aditivo reduz a quantidade de água em relação ao cimento, o que é responsável pelo grande aumento de resistência à compressão dos concretos (AїTCIN, 1995).

O CAD apresenta elevada resistência à compressão comparado ao concreto convencional, baixa permeabilidade, boa trabalhabilidade, alta durabilidade e ótima aderência sobre os concretos pré-existentes. Além disso, o CAD possibilita redução das dimensões das peças estruturais (principalmente pilares), maior velocidade de execução, diminuição das cargas das fundações, aumento do espaço útil de pavimentos inferiores, entre outros.

O metacaulim é produzido através da matéria prima caulim, um mineral existente em algumas regiões do país. Carmo et al. (2008) diz que o metacaulim foi fabricado primeiramente por calcinação de argilas cauliníticas, e recentemente essa pozolana vem sendo obtida a partir da moagem e calcinação de argilas especiais (caulim de alta pureza), em baixas temperaturas, sendo um material de alta atividade pozolânica.

Largamente utilizado na Europa, Ásia e Estados Unidos, o metacaulim é uma adição mineral de alta eficácia para concretos e produtos à base de cimento Portland, podendo inclusive ser utilizado em argamassas para revestimento (BELTRÃO e ZENAIDE, 2010).

No Brasil, a produção atual de caulim é estimada em torno de 0,8 milhões de toneladas por ano, sendo suas principais utilizações como matéria prima na indústria cerâmica e na produção de papel (NITA, 2006).

Nita (2006) diz que o uso do metacaulim como aditivo mineral ou como material substituto do cimento em concretos e argamassas vem crescendo, resultado de uma ótima atividade como material pozolânico, quando este é processado sob condições adequadas.

Segundo Altair Santos (2012) ainda existem poucas empresas destinadas à extração do caulim e à fabricação do metacaulim, mas o mercado brasileiro está entre os cinco maiores do mundo para o metacaulim. Santos ainda estima que $75 \%$ das empresas relacionadas à produção de cimento e concreto no país utilizam esse material pozolânico.

Comparando os impactos ambientais gerados na produção do cimento e do metacaulim, fica claro que os impactos gerados na fabricação do metacaulim são de menores proporções. 


\section{ANAP

Assim, a sua utilização como uma adição mineral, em substituição parcial do cimento em concretos e argamassas acarretará em menor demanda de cimento, e consequentemente amenizará os impactos ambientais.

\section{OBJETIVOS}

A pesquisa teve como objetivo o estudo da adição de metacaulim em concretos de alto desempenho, para se analisar o comportamento do CAD com relação as resistências à compressão e à tração nas idades 7, 28, 60 e 90 dias, a absorção de água na idade de 28 dias e módulo de elasticidade também nas idades $7,28,60$ e 90 dias. Tais resultados foram comparados com o concreto convencional, sem qualquer adição.

Para tal finalidade foram executados traços de concreto 1:4,68; teor de argamassa de 46,7\%; slump 180 a 220 mm, utilizando teor de substituição de metacaulim de 5, 10, 15 e 20\% em relação à massa de cimento, a fim de se analisar a melhor dosagem a ser utilizada.

\section{METODOLOGIA}

Os materiais utilizados durante a pesquisa estão listados a seguir:

- cimento Portland CP V ARI;

- areia;

- brita basáltica;

- água de abastecimento público;

- metacaulim HP Ultra;

- sílica ativa e

- aditivo químico superplastificante Sika ViscoCrete 5800 FTN.

A dosagem dos materiais foi baseada no método IPT/EPUSP, que possibilitou determinar a quantidade de material utilizada no projeto (HELENE e TERZIAN, 1993).

O processo de mistura dos materiais ocorreu em uma betoneira. Após a mistura, o concreto foi submetido ao Slump test ou Teste de abatimento, um indicador do método de adensamento adequado mediante a altura de queda do concreto, e foi realizado para analisar as condições de trabalhabilidade do concreto utilizado, de acordo com a norma NBR NM67: 1998. Nesta pesquisa a altura de queda utilizada foi $200 \pm 20 \mathrm{~mm}$.

Já o adensamento do concreto nas fôrmas de dimensões $100 \times 200 \mathrm{~mm}$ foi feito por meio de mesa vibratória.

Os capeamentos dos corpos-de-prova foram realizados com uma mistura de enxofre e pozolanas, aquecida em uma panela com temperatura média de $130^{\circ} \mathrm{C}$.

A cura dos corpos-de-prova foi realizada em imersão, com adição de cal a água do tanque para que os CPs não perdessem suas propriedades mecânicas. 


\section{ANAP

\section{REVISTA C I ENTÍFICA}

A resistência à compressão simples dos concretos produzidos foi determinada de acordo com a NBR 5739.

A resistência à tração dos corpos-de-prova foi determinada de acordo com a NBR 7222. Neste ensaio aplica-se uma compressão diametral na geratriz do corpo-de-prova.

A absorção de água do CAD foi obtida por meio do peso seco e do peso saturado do concreto.

O ensaio de módulo de elasticidade foi realizado seguindo a NBR 8522, em que o corpo-deprova foi submetido a um carregamento axial, em uma prensa, e as suas deformações foram medidas através de um extensômetro mecânico-defletômetro.

\section{RESULTADOS}

\subsection{RESISTÊNCIA À COMPRESSÃO}

Com a Figura 1 mostrada é possível observar que com a adição de metacaulim até a porcentagem de $15 \%$ houve um aumento na resistência mecânica do CAD. Isto ocorre devido ao melhor empacotamento das partículas realizada pela substituição de parte do cimento por metacaulim, o que gera um maior arranjo da mistura e uma menor capilaridade, auxiliando na resistência mecânica, já que a porosidade e a capilaridade criam regiões de fragilidade para o concreto. A presença de atividade pozolânica no metacaulim consegue suprir a ausência de parte do cimento, mantendo e melhorando as propriedades mecânicas do concreto.

No entanto houve uma queda na resistência do concreto com $20 \%$ de metacaulim. Isso ocorreu devido ao uso de maior quantidade de aditivo para atingir uma boa trabalhabilidade e devido ao fato de o metacaulim não conseguir suprir as propriedades que o cimento traz ao concreto, obtendo então menores tensões de resistência à compressão para o concreto com porcentagens de substituição do cimento por metacaulim acima de $15 \%$. 


\section{ANAP

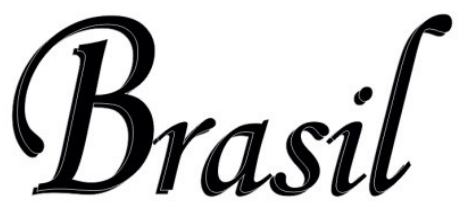

\section{REVISTA C IENTÍFICA}

ficou praticamente constante. Este fato indica que o metacaulim reage na mistura do concreto com o passar do tempo, nos teores indicados, aumentando suas propriedades mecânicas.

Figura 2: Absorção do CAD para diferentes substituições de cimento por metacaulim.

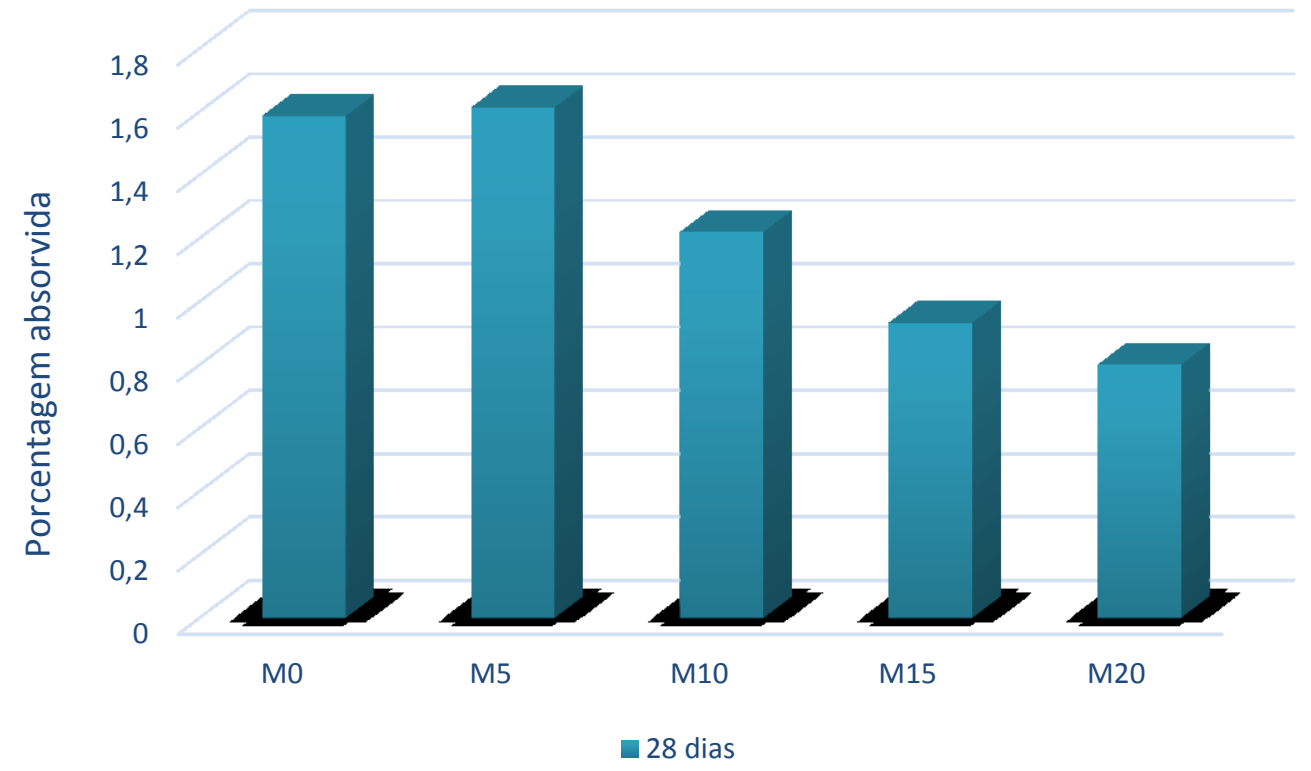

Fonte: Elaborado pelo autor.

O termo MO da Figura representa o CAD com 0\% de substituição de cimento por metacaulim, ou seja, é o traço referência ou controle. Já M5, M10, M15 e M20 representam, respectivamente, os traços de CAD com 5, 10, 15 e $20 \%$ de substituição do cimento por metacaulim.

\subsection{RESISTÊNCIA À TRAÇÃO}

Com base na Figura 3, pode-se notar que com a adição de metacaulim até a porcentagem de $10 \%$ houve um aumento na resistência à tração do CAD. Isso se deve, assim como no caso da resistência à compressão, ao melhor empacotamento das partículas, fato proporcionado pela substituição de parte do cimento pelo metacaulim. Além disso, gera melhor arranjo da mistura e menor capilaridade, diminuindo as regiões de fragilidade do concreto.

A queda na resistência à tração começou no traço de $15 \%$ de substituição, devido ao uso de maior quantidade de aditivo para atingir a trabalhabilidade desejada e também pelo fato de o metacaulim não conseguir suprir as propriedades que o cimento traz ao concreto.

Assim como na compressão, a resistência à tração também apresentou os melhores resultados com o teor de $10 \%$ de substituição de cimento por metacaulim. O traço com essa porcentagem de substituição apresentou as melhores respostas, tendo o metacaulim conseguido suprir a 


\section{ANAP v. 9, n. 17}

\section{REVISTA CIENTÍFICA}

falta de parte do cimento e trouxe ainda melhorias como o aumento da resistência, sem causar danos ao traço.

Figura 3: Resistência à tração do CAD com diferentes substituições de cimento por metacaulim.

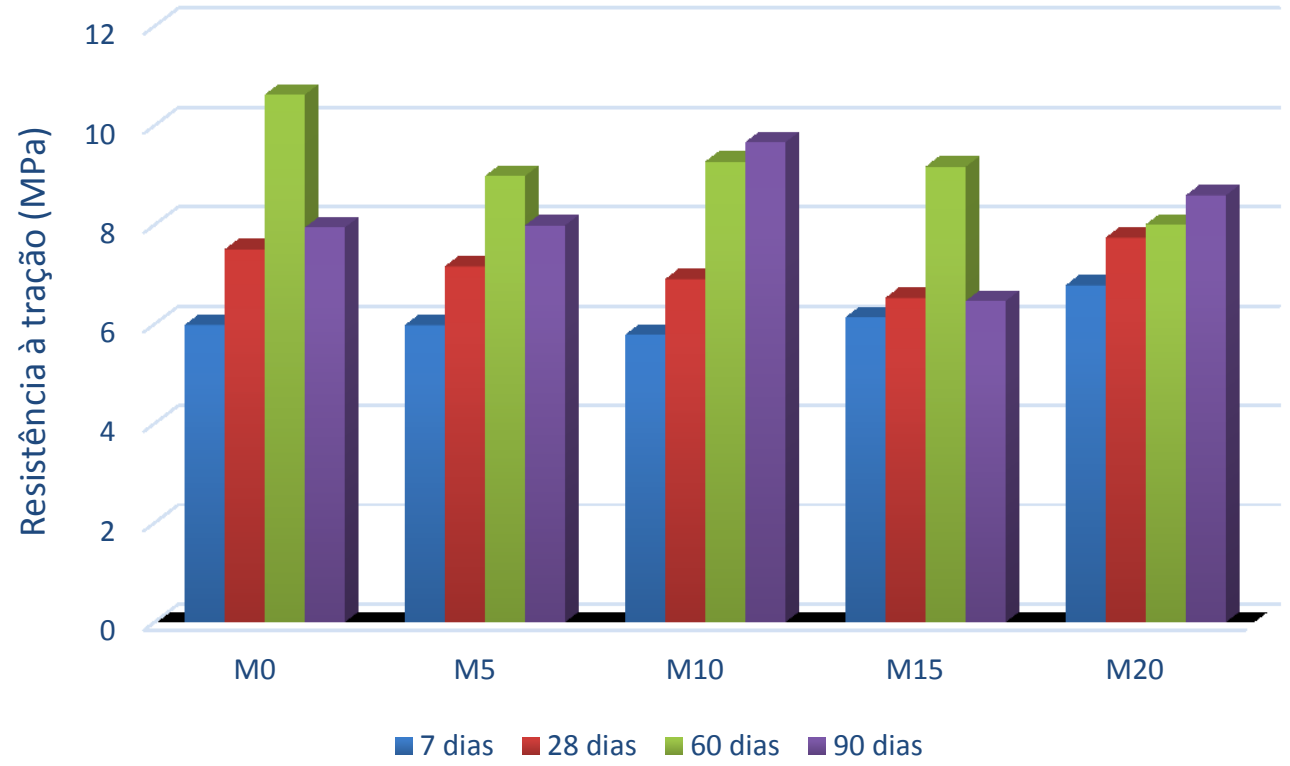

Fonte: Elaborado pelo autor.

O termo MO presente na Figura representa o traço de CAD com $0 \%$ de substituição de cimento por metacaulim, ou seja, é o CAD referência ou controle. Já M5, M10, M15 e M20 representam, respectivamente, os traços de CAD com 5, 10, 15 e 20\% de substituição do cimento por metacaulim.

\subsection{MÓDULO DE ELASTICIDADE}

Através da Figura 4, pode-se notar que o módulo de elasticidade do concreto apresentou pouca variação, independentemente da idade do concreto, conforme o aumento da porcentagem de metacaulim adicionada ao traço.

O ACl 363R-92 (2001) relata que os valores de módulo de elasticidade para o CAD estariam na faixa de 31 a $45 \mathrm{GPa}$, dependendo do método de ensaio utilizado. Contudo, esses valores podem chegar até 50 a 55 GPa (ALMEIDA, 1996b; GIACCIO e ZERBINO, 1996; SILVA, 2000). Assim, pode-se afirmar que os valores encontrados para o módulo de elasticidade encontramse dentro do esperado. 


\section{ANAP

\section{REVISTA CIENTÍFICA}

Figura 4: Módulo de elasticidade do CAD com diferentes substituições de cimento por metacaulim.

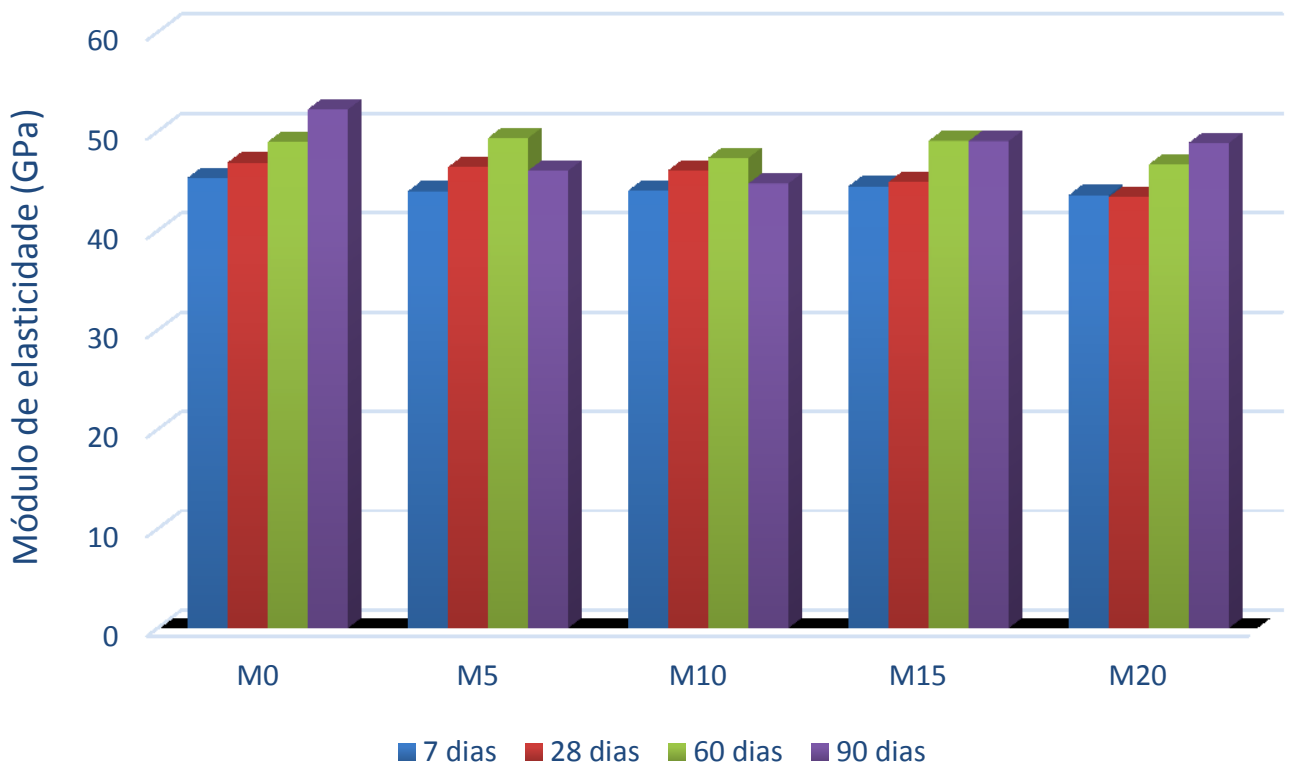

Fonte: Elaborado pelo autor.

O termo MO contido na Figura representa o CAD com $0 \%$ de substituição de cimento por metacaulim, ou seja, é o traço referência ou controle. Já M5, M10, M15 e M20 representam, respectivamente, os traços de CAD com 5, 10, 15 e 20\% de substituição do cimento por metacaulim.

\section{CONCLUSÃO}

Com os resultados dos ensaios de compressão, analisou-se que houve aumento da resistência à compressão do concreto com o aumento de substituição de cimento por metacaulim até $15 \%$. Com $20 \%$, o metacaulim não conseguiu suprir a falta de cimento.

Para o ensaio de absorção, observou-se que o aumento do teor de metacaulim gerou diminuição de cerca de metade do valor da absorção de água para o CAD com $20 \%$ de substituição. Os valores de resistência à tração por compressão diametral dos corpos-de-prova de concreto se mostraram mais efetivos para a adição de $10 \%$ de metacaulim.

Quanto aos valores de módulo de elasticidade, nota-se um ligeiro aumento ao longo do tempo, sendo observado, em geral, reduções nos valores de módulo para aumentos das porcentagens de metacaulim.

Assim, é possível afirmar que o teor de metacaulim de $10 \%$ obteve os melhores resultados. 


\section{ANAP

O metacaulim se torna um ótimo meio para a obtenção de concretos com altos desempenhos, tanto mecânicos, quanto de durabilidade do concreto. Como o produto ainda é novo no Brasil, o custo para se fazer concretos com adição ou substituição de cimento por metacaulim é mais alto que os concretos convencionais, no entanto seu consumo vem aumentando ano a ano, e acredita-se que seu custo irá reduzir tornando-se ainda mais viável a sua utilização para a confecção de concretos. Como mostrado nesta pesquisa com substituição de $10 \%$ de cimento por metacaulim chegamos a valores bem melhores que os encontrados sem sua presença.

\section{AGRADECIMENTO}

Agradeço ao CNPq e ao Grupo MAC - Materiais Alternativos da Construção da UNESP Ilha Solteira, por possibilitarem e viabilizarem a pesquisa.

\section{REFERÊNCIAS BIBLIOGRÁFICAS}

AïTCIN, P. C. Concreto de alto desempenho. São Paulo: Pini, 2000.

AїTCIN, P. C. Developments in the application of high-performance concretes. Construction and building materials, v. 9, n. 1, p. 13-17, 1995.

ALMEIDA, I. R. A influência da microssílica e do superplastificante na durabilidade dos concretos. In: Seminário $O$ Concreto - A utilização da sílica ativa em sua composição, 1., Rio de Janeiro, Fevereiro, 1996b.

AMERICAN CONCRETE INSTITUTE. Committee 363. State-of-the-art report on high-strength concrete, ACI $363 \mathrm{R}-92$ (Reapproved 1997). ACI Manual of Concrete Practice 2001. ACl, Detroit (USA), 2001. 55p.

ASSOCIAÇÃO BRASILEIRA DE NORMAS TÉCNICAS. NBR 5739 - Concreto - Ensaios de compressão de corpos-deprova cilíndricos. Rio de Janeiro, 2007.

ASSOCIAÇÃO BRASILEIRA DE NORMAS TÉCNICAS. NBR 7215 - Cimento Portland - Determinação da Resistência à Compressão. Rio de Janeiro, 1996.

ASSOCIAÇÃO BRASILEIRA DE NORMAS TÉCNICAS. NBR 7222 - Concreto e argamassa - Determinação da resistência à tração por compressão diametral de corpos-de-prova cilíndricos. Rio de Janeiro, 2011.

ASSOCIAÇÃO BRASILEIRA DE NORMAS TÉCNICAS. NBR 8522 - Concreto - Determinação do módulo estático de elasticidade à compressão. Rio de Janeiro, 2008.

ASSOCIAÇÃO BRASILEIRA DE NORMAS TÉCNICAS. NBRNM 67 - Concreto - determinação da consistência pelo abatimento do troco de cone. Rio de Janeiro, 1998.

BELTRÃO, F. C. M.; ZENAIDE, J. C. A influência do Metacaulim nas propriedades do concreto, 2010. Belém.

CARMO, J. B. M.; PORTELLA, K. F. Estudo comparativo do desempenho mecânico da sílica ativa e do metacaulim como adições químicas minerais em estruturas de concreto. Cerâmica, v. 54, n. 331, p. 309-318, 2008. 


\section{ANAP

GIACCIO, G.; ZERBINO, R. Effect of aggregate type on the behavior of high strength concrete. In: INTERNATIONAL CONGRESS ON HIGH-PERFORMANCE CONCRETE, AND PERFORMANCE AND QUALITY OF CONCRETE STRUCTURES, 1996, Florianópolis. Proceedings... Florianópolis, SC, Jun. 1996. P. 52-60.

HELENE, P.; ANDRADE, T. Concreto de cimento Portland. Materiais de Construção Civil e Princípios de Ciência e Engenharia dos Materiais. São Paulo: IBRACOM, 2007.

HELENE, P.; TERZIAN, P. Manual de dosagem e controle de concreto, 1993. PINI, São Paulo, 349p.

MINISTÉRIO DO MEIO AMBIENTE - Cidades sustentáveis, urbanismo sustentável, construção sustentável. Disponível em: <http://www.mma.gov.br/cidades-sustentaveis/urbanismo-sustentavel/constru\%C3\%A7\%C3\%A3osustent\%C3\%A1vel> Acesso em: 15 agosto 2016.

NEVILLE, A. M. Propriedades do concreto. 2. ed. São Paulo: Pini. 1997.

NITA, C. Utilização de pozolanas em compósitos de cimento reforçados com fibras de celulose e PVA. 2006.

QUÍMICA ENSINADA - Cimento ecológico remove gás carbônico. Disponível em: <http://quimicaensinada.blogspot.com.br/2011/11/cimento-ecologico-remove-gas-carbonico.html> Acesso em: 15 agosto 2016.

SANTOS, A. Aliado do cimento, Metacaulim expande mercado no Brasil, 2012. Disponível em: < http://www.cimentoitambe.com.br/aliado-do-cimento-metacaulim-expande-mercado-no-brasil/>. Acesso em: 16 agosto 2016.

SILVA, I. J. Contribuição ao estudo dos concretos de elevado desempenho com adição de sílica ativa: propriedades mecânicas, durabilidade e características da microestrutura. São Paulo, 2000. 266p. Tese (Doutorado em Engenharia Civil) - Escola de Engenharia de São Carlos - Universidade de São Paulo.

Sindicato Nacional da Indústria do Cimento - SNIC. Relatório Anual até 2013. Disponível em: <http://www.snic.org.br/pdf/RelatorioAnual2013final.pdf>. Acesso em: 15 agosto 2016.

TOLEDO FILHO, R. D. et al. Desenvolvimento de concretos de baixo impacto ambiental utilizando materiais cimentícios de baixo consumo de energia e emissão de CO2. Rio de Janeiro: CBE, 2002.

VALVERDE, F. M. Agregados para a construção civil. Balanço mineral brasileiro, 2001. 